\title{
Satisfacción laboral en tiempos de Covid-19 en colaboradores de gobiernos locales de Arequipa
}

\author{
Bellido Medina Rildo Santos \\ ORCID: https://orcid.org/0000-0002-8699-3490 \\ rbellidome@unsa.edu.pe \\ Universidad Nacional de San Agustín de Arequipa \\ Arequipa - Perú \\ Gamarra Castellanos María Elena \\ ORCID: https://orcid.org/0000-0001-6296-3601 \\ mgamarrac@unsa.edu.pe \\ Universidad Nacional de San Agustín de Arequipa \\ Arequipa - Perú
}

\author{
Morales Palao Blanca \\ ORCID: https://orcid.org/0000-0001-9204-3400 \\ bmorales@unsa.edu.pe \\ Universidad Nacional de San Agustín de Arequipa \\ Arequipa - Perú \\ Calizaya López José Manuel \\ ORCID: https://orcid.org/0000-0001-6221-0909 \\ jcalizayal@unsa.edu.pe \\ Universidad Nacional de San Agustín de Arequipa \\ Arequipa - Perú
}

Recibido (04/02/21), Aceptado (23/02/21)

Resumen: Se estudió la satisfacción laboral en tiempos de Covid-19 en colaboradores de gobiernos locales según variables sociolaborales. Se exponen los elementos más relevantes que permiten caracterizar la situación laboral actual de la población en dos gobiernos locales de la ciudad de Arequipa- Perú. Para el análisis se consideró una muestra de 500 participantes, y se utilizó un instrumento de recolección de información con 27 ítems que hicieron posible la comprensión de las exigencias laborales y de la satisfacción en el trabajo de las personas. Los resultados muestran que existe regular satisfacción laboral con tendencia a la insatisfacción durante el año 2020, además, las condiciones de trabajo y el temor al contagio han impactado considerablemente en la conducta de las personas y sus diversas problemáticas familiares.

Palabras Clave: Satisfacción laboral, Covid-19, gobiernos locales, colaboradores.

\section{Job satisfaction in times of Covid-19 in Workers of local governments from Arequipa}

\begin{abstract}
Job satisfaction in times of Covid-19 was studied in local government employees according to socio-labor variables. The most relevant elements that allow characterizing the current employment situation of the population in two local governments of the city from Arequipa-Peru are exposed. For the analysis, a sample of 500 participants was considered, and an information collection instrument with 27 items was used that made it possible to understand people's work demands and job satisfaction. The results show that there is regular job satisfaction with a tendency to dissatisfaction during the year 2020, in addition, working conditions and the fear of contagion have had a considerable impact on the behavior of people and their various family problems.
\end{abstract}

Keywords: Job satisfaction, Covid-19, local governments, workers. 


\section{INTRODUCCIÓN}

Las disposiciones sanitarias en materia laboral dispuestas por el Gobierno Peruano para evitar la propagación de los contagios por el Covid-19 señalan el distanciamiento y aislamiento social por lo cual, los colaboradores deberían de realizar sus actividades a través del teletrabajo de acuerdo con el puesto de trabajo y en otros casos acudir al centro laboral, pero con todas las medidas preventivas y de seguridad correspondientes.

Según la Encuesta Nacional de Hogares para el segundo trimestre 2020 [1], más de 6 millones de personas perdieron su empleo, se incremento la tasa de desempleo en hombres, lo que demuestra que la calidad de empleo se perturbo, si bien a partir de junio se reinicio las actividades laborales estas se dieron bajo otras condiciones que perjudicaron a los colaboradores $19 \%$ trabajo desde su hogar, $17 \%$ salió a trabajar con autorización y $8 \%$ no tenía una autorización formal.

En estos tiempos, Bonilla, Plaza, Soacha y Riaño-Casallas [2] con la aparición e inclusión de fenómenos ocasionados por la pandemia, surgen aspectos importantes tales como son las nuevas formas de relaciones laborales, organización del trabajo y condiciones de trabajo. Todos estos nuevos procesos generan en los trabajadores nuevos riesgos, que implican la aparición de enfermedades laborales, o el desencadenamiento de accidentes de trabajo, lo que tendrá un impacto directo en la percepción de la satisfacción con estos elementos, modificando las actitudes de los trabajadores. Además, Peraza [3] menciona que la salud de los trabajadores es un requisito esencial para los ingresos familiares, la productividad y el desarrollo económico de una nación, siendo, sin dudas, un tema que preocupa a todos, y que cuenta con matices diferentes en cada país, incidiendo directamente en las actitudes que presentan los trabajadores en estas nuevas condiciones laborales, que tienen un impacto directo en la satisfacción laboral.

A partir de lo descrito, se vio por conveniente realizar el estudio en este tipo de organizaciones públicas con el objetivo de analizar la satisfacción laboral en tiempos de Covid-19 en colaboradores de gobiernos locales según variables sociolaborales. Siendo un estudio transversal, expost facto, no experimental [4], así mismo, el tiempo de recolección de información se realizó en los meses de julio y agosto del presente año.

\section{DESARROLLO}

Tal como lo señalan Pujol y Dabos [5] desde los inicios del siglo XX, ha existido un interés muy notorio de la comunidad científica por indagar los factores que determinan la satisfacción laboral, oscilando los estudios considerados como más destacados entre aquellos que se encuentran más fuertemente centrados en las características de la situación, denominados situacionalistas, y los centrados en las características del individuo, llamados disposicionalistas.

En ese sentido, Yáñez, Arenas y Ripoll [6] señalan que la satisfacción laboral es una de las variables laborales estudiadas con mayor frecuencia en lo que respecta a los estudios en la investigación de la conducta de los seres humanos en la organización y su relevancia se vincula con el impacto que posee sobre otras importantes variables organizacionales, que en algunos casos pueden ser consideradas como determinantes del éxito organizacional. Además, Chiang y San Martin [7] consideran que la satisfacción laboral es, básicamente, un concepto globalizador con el que se hace referencia a las actitudes de las personas hacia diversos aspectos de su trabajo, por lo tanto, la satisfacción laboral implica abordar el tema de las actitudes.

De la misma manera investigadores como Chian y Ojeda [8] señalan que la actitud se considera como la evaluación de una situación de experiencia, valorándolo como bueno o malo, agradable o desagradable, deseable o indeseable, por ello, que se trata de una estimación que afecta a la esfera del pensamiento, pero también a los sentimientos y a la determinación comportamental de la persona [9]. En ese sentido, la satisfacción laboral se puede relacionar directamente con el conocimiento de la persona dentro de una organización, esta experiencia vivida se convierte en la percepción del trabajador, y esta última culmina en un componente emocional.

Por otro lado, para Arias y Arias [10] la satisfacción laboral es una respuesta emocional positiva al puesto laboral, que resulta de la evaluación de si el puesto cumple o permite cumplir los valores laborales del trabajador. Así mismo, [11] la satisfacción laboral se centra en la actitud frente a las experiencias laborales, no existiendo un modelo o estrategia concreto para lograr o alcanzar la satisfacción en el trabajador, esto depende de cada organización a través de un sistema administrativo que le permita cumplir con los objetivos personales y profesionales de los integrantes de la organización [12].

Además, la satisfacción laboral está compuesta por varios factores, por un lado, depende de la dificultad de la tarea asignada o por la simplicidad de esta, también se pueden identificar o relacionar con otros factores como el vínculo con el supervisor, las relaciones interpersonales, y el ambiente laboral. Estos factores se relacionan con la experiencia de las personas dentro del entorno organizacional [13], de igual manera, esta experiencia se convierte en la percepción del trabajador, en base a sus conocimientos cognitivos que culminan en el componente emocional, que genera o desemboca 
en el comportamiento o en la manera de proceder del trabajador.

Es por ello que, las dimensiones de la satisfacción laboral se centran en el grado de complacencia, gusto, aprecio, agrado por el trabajo, dependiendo de factores intrínsecos y extrínsecos como, las condiciones laborales, los beneficios, las políticas institucionales, las relaciones sociales, el desarrollo personal, las tareas y la relación con los superiores [14].

En el Perú, Boada [15] señala que existe un enfoque multidimensional para evaluar la satisfacción laboral, para lo cual considera un conjunto de factores, que pueden ser medidos con el fin de calcular el nivel de satisfacción laboral del trabajador, así también, menciona un enfoque unidimensional en el que considera a la actitud como un factor general del individuo con su trabajo, incluso, el considerar evaluar periódicamente la satisfacción percibida por los trabajadores, considera diferentes aspectos de la satisfacción, incluyendo la significación de la tarea, así como, intervenir en caso de insatisfacción laboral, para motivar al trabajador en este aspecto y poder corregir su comportamiento en el ejercicio de sus funciones.

Por lo tanto, de acuerdo con los estudios realizados en el sector público sobre satisfacción laboral se señala que la modernización del Estado es fundamental para que sus instituciones y organizaciones funcionen eficientemente a través de la implementación de sistemas de trabajo y sobre todo la participación de sus colaboradores valorados, reconocidos y con condiciones laborales de calidad [16].

\section{METODOLOGÍA}

Se incluyeron 511 colaboradores de 2 Municipios de gestión local de la Ciudad de Arequipa elegidos aleatoriamente, Municipalidad 1 (311 colaboradores) y Municipalidad 2 (200 colaboradores), el rango de edad de los colaboradores se encuentra entre los 20 a 59 años, el $32.9 \%$ fueron mujeres y el $67.1 \%$ hombres. $31.5 \%$ mantenían la condición laboral de nombrados, 43.1\% contratados y $25.4 \%$ CAS.

El instrumento utilizado fue la escala de satisfacción laboral SL- SPC [17]. La escala evalúa el nivel de satisfacción laboral según 7 factores, Factor I. Condiciones físicas y/o materiales (5 ítems), Factor II. Beneficios Laborales y /o remunerativos (4 ítems), Factor III. Políticas administrativas (5 ítems), Factor IV. Relaciones sociales (4 ítems), Factor V. Desarrollo personal (6 ítems), Factor VI. Desempeño de tareas (6 ítems) y Factor VII. Relación con la autoridad (6 ítems). Los valores de la escala se encuentran entre 1 y 5 de respuesta tipo Likert donde 1 es "totalmente en desacuerdo" y 5 es "totalmente de acuerdo", el puntaje total resulta de sumar las puntuaciones alcanzadas en las respuestas de cada ítem, el puntaje oscila entre 36 y 180, los puntajes altos significan "satisfacción frente al trabajo" y los puntajes bajos "insatisfacción frente al trabajo", existe la posibilidad de obtener puntajes parciales por factores. Además, en el instrumento se adicionaron características sociolaborales como edad, sexo, condición laboral y centro de trabajo, así mismo, se consignaron los datos del consentimiento informado. Para la versión a la muestra local se obtuvieron los niveles de confiabilidad de la escala mediante el método de consistencia interna con la prueba alfa de Cronbach, por lo tanto, el instrumento posee buena confiabilidad cuando los valores (alfa) $\alpha \geq 0.700$ [18] para el caso se obtuvo un $\alpha=$ 0.795 de acuerdo con la medición el alfa es aceptable.

Para la aplicación del instrumento inicialmente, se solicitó la autorización a las autoridades de los gobiernos locales, luego el instrumento fue adaptado al formato Google forms y enviado a los correos electrónicos de los colaboradores previa información del objetivo de la investigación, las instrucciones de la escala y la confidencialidad de los datos proporcionados, aceptando participar voluntariamente firmando el consentimiento informado respectivo.

\section{RESULTADOS}

Los resultados de la investigación comparan el nivel de satisfacción laboral según factores sociolaborales como sexo, edad, centro de trabajo y condición laboral, por lo tanto, se exploraron los datos para analizar la distribución, la asimetría, curtosis y pruebas de normalidad evidenciando que no existe distribución normal ( $\mathrm{p}<$ $.05)$. Se realizó el análisis descriptivo y el comparativo de la satisfacción laboral según sexo, grupo etario, condición laboral y centro de trabajo. Para comparar dos muestras independientes se utilizó la U de Mann-Whitney siendo su tamaño del efecto (TE) calculó de la probabilidad de superioridad (PSest) las normas interpretativas, No efecto (PSest $\leq=0.0$ ), pequeño (PSest $\geq$ $0,56)$, mediano (PSest $\geq 0,64)$ y grande (PSest $\geq 0,71$ ) [19] [20]. La comparación de más de dos muestras independientes se realizó con la $\mathrm{H}$ de Kruskal Wallis, su tamaño del efecto utilizado fue $(\mathrm{n} 2 \mathrm{H})$ eta cuadrado [21] siendo sus normas interpretativas $(\mathrm{n} 2 \mathrm{H} \geq 0.04$ mínima necesaria), ( $\mathrm{n} 2 \mathrm{H} \geq 0.25$ moderada), $(\mathrm{n} 2 \mathrm{H} \geq 0.64$ fuerte) [22], y para establecer las correlaciones, se empleó la Rho de Spearman. Utilizándose para el análisis estadístico el programa SPSS 25.0 y JASP. 


\section{NIVEL DE SATISFACCIÓN LABORAL}

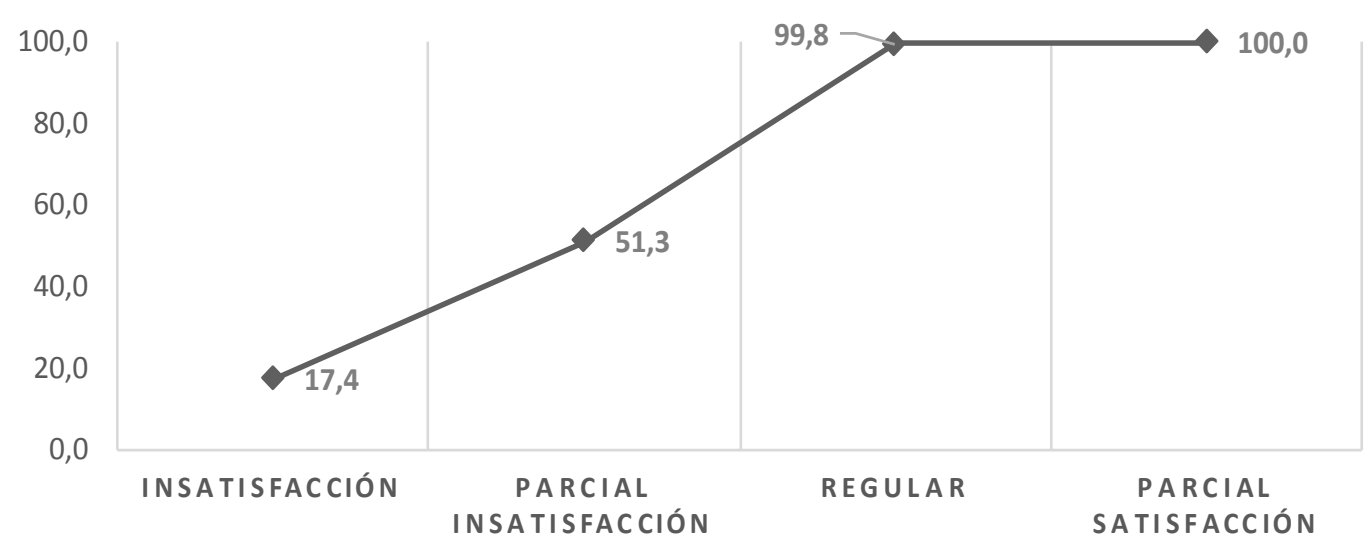

Figura 1. Nivel de satisfacción laboral: Ojiva Acumulada para definir el nivel de satisfacción laboral

En la figura se observa los datos del nivel de satisfacción laboral que presentan los colaboradores de los gobiernos locales, describiendo que el $99.8 \%$ de los encuestados se encuentra en el nivel regular con tendencia a la insatisfacción frente al trabajo, siendo los factores extrínsecos hacia el colaborar los que más inciden en este nivel sobre todo por las nuevas condiciones laborales que se han establecido resultado de la pandemia (en algunos casos el teletrabajo, reducción de remuneraciones en la mayoría de colaboradores y la obligación de realizar actividades presenciales exponiéndose al contagio generando temor).

TABLA 1. Valores numéricos del nivel de satisfacción laboral

\begin{tabular}{lccccc}
\hline \multicolumn{1}{c}{ Nivel } & $M$ & $D E$ & $M d$ & Min. & Máx. \\
\hline I. Condiciones fisicas y/o materiales & 16.52 & 3.242 & 17 & 6 & 23 \\
II. Beneficios laborales y/o remunerativos & 11.64 & 2.116 & 11 & 6 & 19 \\
III. Políticas administrativas & 13.09 & 2.735 & 13 & 7 & 25 \\
IV. Relaciones sociales & 11.92 & 2.632 & 13 & 4 & 19 \\
V. Desarrollo personal & 18.91 & 4.583 & 21 & 6 & 26 \\
VI. Desempeño tareas & 17.48 & 3.902 & 18 & 6 & 29 \\
VII. Relación con la autoridad & 17.47 & 3.714 & 18 & 6 & 29 \\
\hline
\end{tabular}

Nota: $\mathrm{M}=$ Media; $\mathrm{D}=$ Desviación estándar; $\mathrm{Md}=$ Mediana; Min. = mínimo; Máx. = máximo

En la tabla se presenta los resultados del nivel de satisfacción laboral que presentan los colaboradores según dimensiones de estudio, de acuerdo con la dimensión condiciones físicas y/o materiales, el trabajo se ha trasladado a los hogares de los colaboradores sin contar con la información y los sistemas de la propia institución para atender la demanda, algún personal se encuentra en la institución, pero sin garantías físicas para asegurar la salud integral exponiéndolo al contagio.

En cuanto a los beneficios laborales y/o remunerativos, el grado de complacencia en relación con el incentivo económico se vio afectado porque a varios colaboradores se les ha reducido su sueldo promedio hasta en un $40 \%$.
En relación con las políticas administrativas, el grado de satisfacción se afectó porque no se han tomado las mejores decisiones para garantizar el trabajo de los colaboradores en algunos casos se despidió personal y en otros se plantearon nuevas medidas que los colaboradores a fin de no perder su trabajo se sometieron.

En la dimensión relaciones sociales, el promedio de regular satisfacción se debe a que los colaboradores ya no interactúan unos con otros y menos colaboran entre ellos para cumplir con los objetivos de la institución.

Según la dimensión desarrollo personal, la regular satisfacción laboral se debe a las políticas institucionales dado que las oportunidades de autorrealización en estos tiempos de pandemia han quedado restringidas 
solo se ha garantizado la continuidad del trabajo para los colaboradores que cuentan con un vínculo laboral estable.

Con respecto a la dimensión desempeño de tareas, el teletrabajo en algunos casos es favorable porque cuentan con las autorizaciones, la información y el sistema para colaborar y en otros es desfavorable porque no cuentan con los recursos y tecnología necesaria para poder cumplir con su trabajo, teniendo un impacto significativo en el nivel de satisfacción laboral.

Y la relación con la autoridad, en estos tiempos por la presencia del Covid-19, no es la más adecuada debido a que los colaboradores sienten que los jefes solo exigen y no valoran el trabajo porque prácticamente pueden ser despedidos en cualquier momento o que se les propongan nuevas condiciones de trabajo.

TABLA 2. Comparación del nivel de satisfacción laboral según sexo

\begin{tabular}{lccccc}
\hline \multirow{2}{*}{\multicolumn{1}{c}{ Dimensiones }} & $\begin{array}{c}\text { Hombres } \\
(n=343)\end{array}$ & $\begin{array}{c}\text { Mujeres } \\
(n=168)\end{array}$ & Estadigrafo & & \multirow{2}{*}{$P S_{\text {est }}$} \\
\cline { 2 - 5 } & Rango & Rango & $U$ & $p$ & \\
\hline Satisfacción laboral & 264.39 & 238.87 & 25934.5 & .066 & 0.45 \\
I. Condiciones físicas y/o materiales & 261.74 & 244.28 & 26843.0 & .206 & 0.47 \\
II. Beneficios laborales y/o remunerativos & 246.06 & 276.30 & 25401.5 & .027 & 0.44 \\
III. Políticas administrativas & 258.14 & 251.63 & 28077.5 & .636 & 0.49 \\
IV. Relaciones sociales & 263.93 & 239.81 & 26091.5 & .078 & 0.45 \\
V. Desarrollo personal & 266.06 & 235.47 & 32261.0 & .027 & 0.56 \\
VI. Desempeño tareas & 263.97 & 239.72 & 26077.0 & .079 & 0.45 \\
VII. Relación con la autoridad & 257.65 & 252.62 & 28244.5 & .716 & 0.49 \\
\hline
\end{tabular}

Nota: $\mathrm{n}=$ tamaño muestral; Rango $=$ Rango Promedio; $\mathrm{U}=\mathrm{U}$ de Mann Withney; $\mathrm{p}=\mathrm{p}$ valor; PSest $=$ Probabilidad de Superioridad (tamaño del efecto).

En base al análisis de las diferencias encontradas en el nivel de satisfacción laboral según sexo, al comparar los datos se identificó que son las mujeres quienes se sienten más insatisfechas en la dimensión beneficios laborales y/o remunerativos, y son los varones quienes se sienten más insatisfechos en la dimensión desarrollo personal (siendo el tamaño del efecto moderado para ambos casos), en relación a las demás dimensiones no se encontraron diferencias en el nivel de satisfacción según hombres y mujeres.

TABLA 3. Comparación del nivel de satisfacción laboral según centro de trabajo

\begin{tabular}{lccccc}
\hline \multirow{1}{*}{\multicolumn{1}{c}{ Dimensiones }} & $\begin{array}{c}\text { Municipio } 1 \\
(n=311)\end{array}$ & $\begin{array}{c}\text { Municipio } 2 \\
(n=200)\end{array}$ & \multicolumn{2}{c}{ Estadígrafo } & \multirow{2}{*}{$P S_{\text {est }}$} \\
\cline { 2 - 5 } & Rango & Rango & $U$ & $p$ & \\
\hline Satisfacción laboral & 322.88 & 152.01 & 10301.0 & .000 & 0.17 \\
I. Condiciones físicas y/o materiales & 329.51 & 141.69 & 8238.0 & .000 & 0.13 \\
II. Beneficios laborales y/o remunerativos & 211.22 & 325.63 & 17173.5 & .000 & 0.28 \\
III. Políticas administrativas & 205.10 & 335.16 & 15269.0 & .000 & 0.25 \\
IV. Relaciones sociales & 311.41 & 169.84 & 13867.5 & .000 & 0.22 \\
V. Desarrollo personal & 336.89 & 130.22 & 5943.0 & .000 & 0.10 \\
VI. Desempeño tareas & 317.10 & 160.99 & 12098.5 & .000 & 0.19 \\
VII. Relación con la autoridad & 319.01 & 158.02 & 11503.0 & .000 & 0.18 \\
\hline
\end{tabular}

Nota: $\mathrm{n}=$ tamaño muestral; Rango = Rango Promedio; $\mathrm{U}=\mathrm{U}$ de Mann Withney; $\mathrm{p}=\mathrm{p}$ valor; PSest= Probabilidad de Superioridad (tamaño del efecto). 
Así también, se compararon los niveles de satisfacción laboral y sus dimensiones de estudio en colaboradores de ambos municipios, se encontraron diferencias estadísticamente significativas en el nivel general y en todas las dimensiones $(\mathrm{p}<.05)$, siendo el tamaño del efecto pequeño según normas interpretativas (menores o iguales PSest $\geq 0,56$ ).

Por lo tanto, los colaboradores del municipio 1 pre- sentan mayor insatisfacción en las condiciones de trabajo, relaciones sociales, desarrollo personal, desempeño de tareas y relación con la autoridad que los colaboradores del municipio 2; y los colaboradores del municipio 2 presentan mayores niveles de insatisfacción en relación con los beneficios y/o remuneraciones laborales y las políticas administrativas en comparación a los colaboradores del municipio 1 .

TABLA IV. Comparación del nivel de satisfacción laboral según condición laboral

\begin{tabular}{llllccc}
\hline \multirow{2}{*}{ Nivel } & $\begin{array}{c}\text { Nombrado } \\
(n=161)\end{array}$ & $\begin{array}{c}\text { Contratado } \\
(n=220)\end{array}$ & \multicolumn{2}{c}{ CAS } \\
& Ran=130) & \multicolumn{2}{c}{ Estadigrafo } & \multirow{2}{*}{$n^{2}{ }_{H}$} \\
\cline { 2 - 5 } & Rango & Rango & Rango & $H$ & $p$ & \\
\hline Satisfacción laboral & 270.26 & 260.78 & 230.26 & 5690 & .058 & 0.01 \\
I. Condiciones físicas y/o materiales & 242.14 & 287.12 & 220.50 & 18986 & .000 & 0.04 \\
II. Beneficios laborales y/o remunerativos & 276.33 & 236.42 & 263.96 & 17500 & .024 & 0.04 \\
III. Políticas administrativas & 286.04 & 222.63 & 275.26 & 20559 & .000 & 0.04 \\
IV. Relaciones sociales & 256.94 & 261.35 & 245.79 & 946 & .623 & 0.00 \\
V. Desarrollo personal & 255.71 & 267.51 & 236.88 & 3560 & .169 & 0.00 \\
VI. Desempeño tareas & 280.76 & 253.05 & 230.33 & 8645 & .013 & 0.01 \\
VII. Relación con la autoridad & 268.77 & 272.13 & 212.88 & 15090 & .001 & 0.04 \\
\hline
\end{tabular}

Nota: $\mathrm{n}=$ tamaño muestral; Rango $=$ Rango Promedio $\mathrm{H}=\mathrm{H}$ de Kruskal Wallis; $\mathrm{p}=\mathrm{p}$ valor. $\mathrm{n} 2 \mathrm{H}=$ eta cuadrada (tamaño del efecto).

Se compararon los niveles de satisfacción laboral y sus dimensiones de estudio según la condición laboral de acuerdo con los datos se precisa que existen diferencias estadísticamente significativas en las dimensiones condiciones físicas y/o materiales, beneficios laborales y remunerativos, políticas administrativas, desempeño de tareas y relación con la autoridad $(\mathrm{p}<.05)$, siendo el tamaño del efecto pequeño.

Así mismo, son los colaboradores contratados quienes manifiestan estar más insatisfechos que los otros colaboradores en relación con las condiciones de trabajo y la relación con la autoridad. Así también, son los colaboradores nombrados quienes presentan mayores niveles de insatisfacción en las dimensiones beneficios laborales, políticas administrativas y desempeño de tareas en comparación a los otros grupos.

\section{CONCLUSIONES}

Los resultados muestran que existe regular satisfacción laboral con tendencia a la insatisfacción durante el año 2020 en los centros laborales, además, las condiciones de trabajo y el temor al contagio han impactado considerablemente en la conducta de las personas y sus diversas problemáticas familiares.

Un aspecto central que genera insatisfacción en los colaboradores en tiempo de Covid-19, es que a las mujeres se les redujo considerablemente el sueldo y a los varones se le cortaron las expectativas de desarrollarse personalmente.

Si bien existen diferencias en las dimensiones del nivel de satisfacción laboral según centros de trabajo la magnitud de esas diferencias es pequeña, es decir, que tanto en el municipio 1 como 2 se presentan similares niveles de insatisfacción debido a que las políticas laborales se modificaron y la situación laboral se percibió afectada por la pandemia Covid-19.

La condición laboral también influye en la insatisfacción laboral tanto en los colaboradores que tienen estabilidad como en los que no la tienen, porque la estabilidad peligra cuando las instituciones carecen de recursos económicos para solventar los sueldos y salarios del personal.

Es fundamental seguir estudiando la satisfacción laboral tanto en las organizaciones del sector público como privado sobre todo en estos contextos difíciles producidos por la pandemia Covid-19, que significativamente afecto la vida laboral de muchos trabajadores del país y del mundo, con la finalidad de entender lo que realmente necesita el colaborador en estos procesos para no cortar sus expectativas.

Finalmente, el estudio es relevante por las características, el contexto y las adversidades actuales, esperando que sea de aporte para las disciplinas científicas que se interesan por el recurso humano en las institucio- 
nes públicas.

\section{REFERENCIAS}

[1]Organización Internacional del Trabajo, «Mi Futuro del Trabajo. Perú: Impacto de la Covid-19 en el empleo y los ingresos laborales,» 28 Septiembre 2020. [En línea]. Available: https:/www.ilo.org/wcmsp5/groups/ public/---americas/---ro-lima/documents/publication/ wcms_756474.pdf. [Último acceso: 10 Octubre 2020]. [2]L. A. Bonilla Prieto, D. C. Plaza Rocha, G. Soacha De Cerquera y M. I. Riaño-Casallas, «Teletrabajo y su Relación con la Seguridad y Salud en el Trabajo,» Ciencia \& trabajo, vol. 16, n 49, pp. 38-42, 2020.

[3]C. X. Peraza de Aparicio, «Salud laboral frente a la pandemia del COVID-19 en Ecuador,» MediSur, vol. 18, no 3, pp. 507-511, 2020.

[4]S. Carrasco, Metodología de la investigación científica. Pautas metodológicas para diseñar y elaborar el proyecto de investigación, Lima: Editorial San Marcos E.I.R.L., 2019.

[5]L. J. Pujol-Cols y G. E. Dabos, «Satisfacción laboral: una revisión de la literatura acerca de sus principales determinantes,» Estudios Gerenciales, vol. 34, $\mathrm{n}^{\mathrm{o}}$ 146, pp. 3-18, 2018.

[6]R. Yañez, M. Arenas y M. Ripoll, «The impact of interpersonal relationships on the general job satisfaction,» Liberabit, vol. 16, n 2, pp. 193-202, 2010.

[7]M. M. Chiang Vega y N. J. San Martín Neira, «Análisis de la satisfacción y el desempeño laboral en los funcionarios de la Municipalidad de Talcahuano,» Ciencia \& trabajo, vol. 17, no 54, pp. 159-165, 2015.

[8]M. M. Chiang Vega y J. F. Ojeda Hidalgo, «Estudio de la relación entre satisfacción laboral y el desempeño de los trabajadores de las ferias libres.,» Contaduría y administración, vol. 58, nº 2, pp. 39-60, 2013.

[9]M. G. Sánchez Trujillo y L. E. García Vargas, «Satisfacción Laboral en los Entornos de,» Scientia et Technica , vol. 22, no 2, pp. 161-166, 2017.

[10]W. L. Arias Gallegos y G. Arias Cáceres, «Relación Entre el Clima Organizacional y la Satisfacción Laboral en una Pequeña Empresa del Sector Privado,» Ciencia \& trabajo, vol. 16, no 51, pp. 185-191, 2014.

[11]A. Omar, «Liderazgo transformador y satisfacción laboral: el rol de la confianza en el supervisor,» Liberabit, vol. 17, n² 2, pp. 129-138, 2011.
[12]J. L. Hernández Juárez, «Evaluación de la motivación y satisfacción laboral en un organismo autónomo de la administración pública del Estado de Sinaloa,» Daena: International Journal of Good Conscience, vol. 12, no 2, pp. 107-147, 2017.

[13]M. G. Abrajan Castro, J. M. Contreras Padilla y S. Montoya Ramírez, "Grado de satisfacción laboral y condiciones de trabajo: una exploración cualitativa,» Enseñanza e Investigación en Psicología, vol. 14, $\mathrm{n}^{\mathrm{o}}$ 1, pp. 105-118, 2009.

[14]D. A. Cernas, P. Mercado y F. León, «Satisfacción laboral y compromiso organizacional: prueba de equivalencia de medición entre México y Estados Unidos,» Contaduría y administración, vol. 63, $\mathrm{n}^{\circ} 2$, pp. 1-23, 2018.

[15]N. A. Boada Llerena, «atisfacción laboral y su relación con el desempeño laboral en una Pyme de servicios de seguridad en el Perú,» ournal of Economics, Finance and International Business, vol. $3, n^{\circ} 1$, pp. 75103, 2019.

[16]M. Chiang, C. M. Salazar, P. Huerta y A. Nuñez, «Clima organizacional y satisfacción laboral en organizaciones del sector estatal (Instituciones públicas) Desarrollo, adaptación y validación de instrumentos,» Universum (Talca), vol. 23, no 2, pp. 66-85, 2008.

[17]S. Palma, Escala de Satisfacción Laboral (SL-SPC) Manual., Lima: Editora CARTOLAN EIRL., 2005.

[18]D. Frías, Apuntes de consistencia interna de las puntuaciones de un instrumento de medida, Valencia: Universidad de Valencia, 2019.

[19]J. L. Ventura, «Tamaño del efecto para la U de Mann-Whitney: aportes al artículo de Valdivia-Peralta et al.,» Revista chilena de neuro-psiquiatría, vol. $54, \mathrm{n}^{\circ}$ 4, pp. 353-354, 2016.

[20]R. J. Grissom, «Probability of the superior outcome of one treatment over another.,» Journal of Applied Psychology, vol. 79, no 2, pp. 314-316, 1994.

[21]M. Tomczak y E. Tomczak, «The need to report effect size estimates revisited. An overview of some recommended measures of effect size,» Trends Sport Sciences, vol. 1, nº 21, pp. 19-25, 2014.

[22]S. Domínguez, «Magnitud del efecto, una guía rápida,» Educación Médica, vol. 19, n 4, pp. 251-254, 2018. 


\section{RESUMEN CURRICULAR}

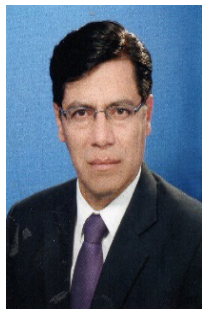

Bellido Medina, Rildo Santos, Doctor en Psicología y Magíster en Gerencia Estratégica de Recursos Humanos. Docente auxiliar en el programa de estudios de Relaciones Industriales, Docente investigador UNSAINVESTIGA y miembro del Instituto de Investigaciones Sociales de la Universidad Nacional de San Agustín, Arequipa, Perú

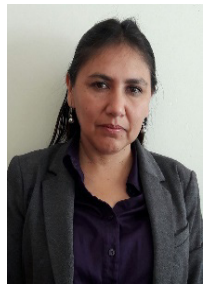

Morales Palao, Blanca, Magíster en Ciencias, Relaciones Industriales con mención en Gerencia Estratégica de Recursos Humanos, Licenciada en Ciencias de la Comunicación, Especialidad Relaciones Públicas, Diplomado en Recursos Humanos, Docente Auxiliar del programa de estudios de Ciencias de la Comunicación y miembro del Instituto de Investigaciones Sociales de la Universidad Nacional de San Agustín, Arequipa, Perú

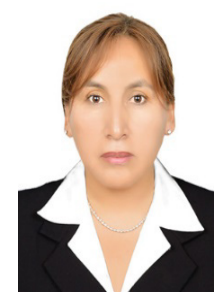

Gamarra Castellanos María Elena, Magíster en Ciencias con mención en Relaciones Industriales con mención en Gerencia Estratégica de Recursos Humanos, Grado de Doctor en Comunicación y Desarrollo; Licenciado en Ciencias de la Comunicación, Especialidad Relaciones Públicas. Docente Auxiliar en el programa de estudios de Relaciones Industriales en la Universidad Nacional de San Agustín, Arequipa, Perú

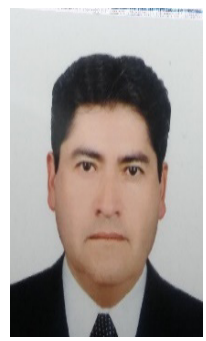

Calizaya López, José Manuel, Doctor en Ciencias de la Educación, Magíster en Ciencias con Mención en Gestión Social - Desarrollo Sostenible y Licenciado en Trabajo Social. Docente Principal y director del Departamento Académico de Trabajo Social, Docente investigador UNSA- INVESTIGA y miembro del Instituto de Investigaciones Sociales de la Universidad Nacional de San Agustín, Arequipa, Perú 